\title{
Producing Squeezed Input States for an Atomic Clock Using an Optical Cavity
}

\author{
Ian D. Leroux, Monika H. Schleier-Smith and Vladan Vuletić \\ Department of Physics, MIT-Harvard Center for Ultracold Atoms, and Research Laboratory of Electronics \\ Massachusetts Institute of Technology, Cambridge, Massachusetts 02139, USA
}

\begin{abstract}
Spin squeezing, the generation of collective states of atomic ensembles with reduced spin noise by exploiting nonclassical correlations between particles, is a promising approach to overcoming the Standard Quantum Limit set by projection noise of independent atoms. We briefly present two successful implementations of spin squeezing in ensembles of ${ }^{87} \mathrm{Rb}$ confined within and optical resonator, and discuss some of the decoherence mechanisms, both technical and fundamental, encountered in our implementation of spin squeezing.
\end{abstract}

\section{INTRODUCTION}

The projection noise of uncorrelated test particles imposes a limit on measurement precision known as the Standard Quantum Limit, relevant to current time and frequency measurements [1]. The use of quantum mechanical entanglement to overcome this limit is an active area of research. In the context of frequency metrology using large atomic ensembles such entanglement is usually discussed in the language of spin squeezing [2]. The "spin" here refers to the collective pseudospin obtained by summing the individual spin- $\frac{1}{2}$ Bloch vectors associated with each two-level atom in the ensemble: $\boldsymbol{S}=$ $\sum_{i} s_{i}$. The "squeezing" indicates the exchange of uncertainty between the two directions orthogonal to the mean spin $\langle\boldsymbol{S}\rangle$; reducing noise in the spectroscopically important direction while increasing it in the other (irrelevant) direction. Spin squeezing has been studied experimentally for over a decade, with demonstrations of squeezing by absorption of squeezed light [3], by entangling operations performed on a few ions [4], or by exploiting repulsive interactions in a BEC in a multiwell potential [5]. Squeezing within the Zeeman manifold of individual atoms with large angular momentum has also been achieved [6], [7] However, it is only in the past year that field-insensitive collective pseudo-spins of large atomic ensembles suitable for precision frequency spectroscopy have been squeezed [8], [9]. In this paper we discuss some of the experimental techniques which have enabled this development and briefly review the results achieved so far.

The system in which we have been exploring spin squeezing for Ramsey spectroscopy consists of an ensemble of laser-cooled ${ }^{87} \mathrm{Rb}$ atoms confined, using a far off-resonant dipole trap, within an optical resonator. We use the canonical $\left|F=1, m_{F}=0\right\rangle \leftrightarrow\left|F=2, m_{F}=0\right\rangle$ clock transition for our effective two-level atom. The resonator is tuned in between the optical resonance frequencies of the two clock states (Figure 1c), such that the index of refraction of an atom in $|\uparrow\rangle \equiv\left|F=2, m_{F}=0\right\rangle$ blue-shifts the resonator frequency

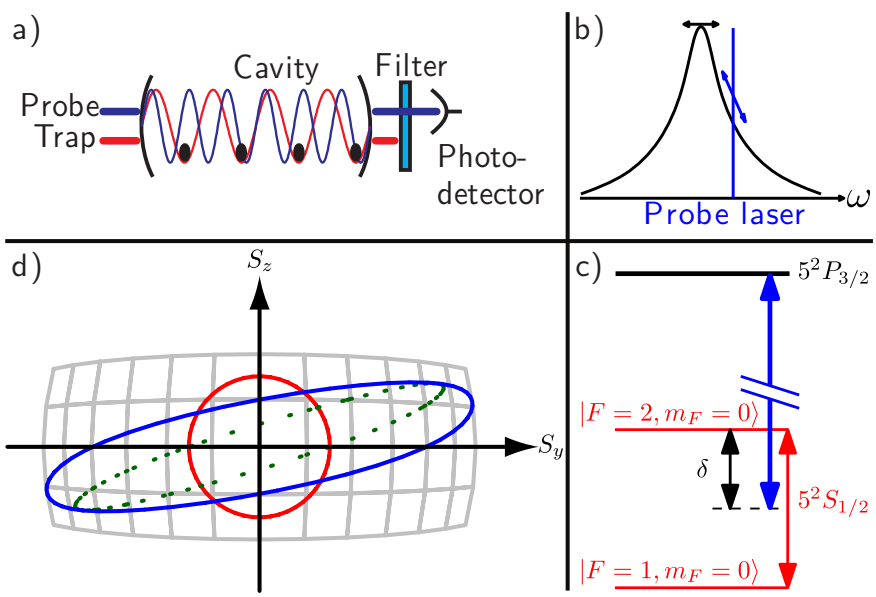

Fig. 1. (a) Setup schematic: the atoms are combined in a 1-D lattice generated by a standing wave of $851 \mathrm{~nm}$ light in the cavity. A probe laser at $780 \mathrm{~nm}$ traverses the cavity and is detected on an avalanche photodiode. (b) The probe laser is detuned by one half-linewidth from cavity resonance, so that the transmitted probe power fluctuates according to the frequency shift of the cavity resonance. (c) Level scheme: The field-insensitive states in the ground-state hyperfine manifold form an effective two-level atom (clock pseudo-spin) with a $6.8 \mathrm{GHz}$ transition frequency (red). The detuning $\delta$ of the cavity resonance (blue) from the atomic optical transition is chosen to be halfway between the optical line centers for the two clock states, such that they are equally coupled to the cavity field but with opposite sign. (d) Deformation of spin uncertainty region by the coupled atom-cavity dynamics (see text). An initially uncorrelated coherent spin state (red circle) is sheared by the $S_{z}$-dependent phase shift into an ellipse (dotted green ellipse). Even after including the increased area of the uncertainty region due to photon shot noise (blue ellipse), the short axis of the ellipse is still narrower than the original diameter of the uncertainty region.

by the same amount that an atom in $|\downarrow\rangle \equiv\left|F=1, m_{F}=0\right\rangle$ red-shifts it. The net shift of the cavity resonance is thus proportional to the population difference between the two states, or to $S_{z}$ in the Bloch sphere representation. A drive laser tuned to the slope of the cavity resonance causes both the transmitted and intracavity powers to vary in proportion to this cavity shift. Monitoring the transmission of this laser reveals the cavity shift and so performs a measurement of $S_{z}$, while the variation of intracavity power acts back on the atoms via the light shift to produce an effective interaction that can also be used for squeezing.

We emphasize that the spin squeezing techniques being studied here are tools for engineering the input state to a Ramsey sequence. All squeezing is to be performed before the Ramsey sequence runs, and no additional operations are 
required during the sequence itself [8], [9]. In particular, the accuracy of the spectroscopy is not affected in principle by any phase shifts discussed in this paper, all of which occur before the initial phase in the clock sequence is specified by the first Ramsey pulse.

In Section II, we review the major decoherence mechanisms that interfere with the realization of spin squeezing in systems such as ours and present some experiental techniques we have found helpful in overcoming them. Section III summarizes the squeezing results themselves, which are presented in more detail in separate publications [9], [10].

\section{DECOHERENCE}

\section{A. Magic-Polarization Trap}

The atoms are confined in a dipole trap created by $851 \mathrm{~nm}$ light traversing the cavity mode (Figure 1a). This is not a magic-wavelength trap, in the sense that the scalar polarizabilities of the two clock states differ by $\frac{\omega_{\mathrm{HF}}}{\left|\Delta_{\text {eff }}\right|}=2.3 \times 10^{-4}$ where $\omega_{\mathrm{HF}}=2 \pi \times 6.834 \mathrm{GHz}$ is the energy difference between the clock states and $\Delta_{\text {eff }}=2 \pi \times-29.3 \mathrm{THz}$ is the effective detuning of the trap light from the optical transition, averaged over the $\mathrm{D}_{1}$ and $\mathrm{D}_{2}$ lines. Thus the trap light shifts and inhomogeneously broadens the clock transition. We overcome this by giving the trap light an elliptical polarization and by introducing a bias field $B_{0}$ along the cavity axis. This allows the vector light shift, which acts as a magnetic field along the beam axis [11], to produce a quadratic Zeeman shift which is linear in local trap intensity and cancels the scalar light shift to linear order:

$$
\begin{aligned}
\frac{\delta(U)}{2 \pi} & =-\frac{\omega_{\mathrm{HF}}}{\Delta_{\text {eff }}} \frac{U}{h}+\beta\left(B_{0}+f b \frac{U}{h}\right)^{2} \\
& =\beta B_{0}^{2}+\left(2 \beta B_{0} f b-\frac{\omega_{\mathrm{HF}}}{\Delta_{\text {eff }}}\right) \frac{U}{h}+\beta\left(f b \frac{U}{h}\right)^{2}
\end{aligned}
$$

where $\delta(U)$ is the trap-induced clock shift, $U$ is the local trap potential, $\beta=574 \mathrm{~Hz} / \mathrm{G}^{2}$ is the quadratic Zeeman coefficient, $f$ is the fraction of circularly polarized light $\left( \pm 1\right.$ for $\sigma^{ \pm}$light, 0 for linearly polarized light) and $b=61 \mathrm{mG} / \mathrm{MHz}$ is the effective magnetic field per unit trap depth for $\sigma^{+}$-polarized trap light. As shown in Figure 2, the cancellation in the second term of Equation 2 can be tuned to make the clock frequency independent of trap light intensity to first order in the vicinity of the mean trap intensity.

The residual broadening, due to the final quadratic term in the equation, becomes smaller as $f$ decreases. Since the cancellation only depends on the product $B_{0} f$, it is advantageous to work at a large bias field and with small polarization fraction. We typically operate at a bias field $B_{0}=5.6 \mathrm{G}$ and with a circular polarization fraction $f=0.5(1)$. For these parameters we find that the residual trap light broadening is $\sim 20 \mathrm{~Hz}$ and, together with the light used to lock the length of the resonator (see next subsection), this limits our coherence time to $\sim 7 \mathrm{~ms}$.

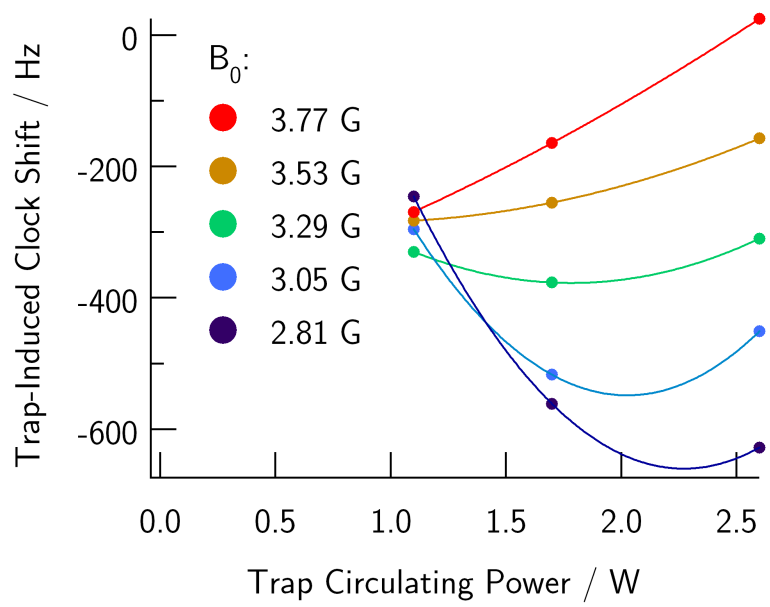

Fig. 2. Cancellation of the trap scalar light shift using the vector light shift. The curves are fits to the mean shift of the clock frequency as a function of trap light intensity for various magnetic bias fields. For appropriate combinations of trap polarization, bias field magnitude and mean trap power, the clock frequency is linearly insensitive to fluctuations in trap intensity. These curves were taken with full circular polarization of the trap light $(f=1)$.

\section{B. Probe Light Dephasing}

The inhomogeneous light shift from the probe light is an altogether more serious problem. The probe light shift is a fundamental consequence of the atom-cavity coupling we use for squeezing. It provides the necessary back-action that preserves the Heisenberg uncertainty relations when we measure $S_{z}$ and is directly useful for squeezing in its own right (see Section III-B). A probe laser that did not cause a light shift on the clock levels (e.g. by operating at a magic wavelength) would not couple to the clock spin state and would be useless for squeezing. Further, the probe intensity inhomogeneity is maximal because the atoms are held in a 1-D optical lattice (the trap light standing wave) which is incommensurate with the standing wave structure of the probe light in the resonator, so that atoms are distributed over all possible couplings from node to antinode (as schematically illustrated in Figure 1a).

The consequence is shown in the blue trace of Figure 3, which is a measurement of the phase imparted to the atomic ensemble by a short pulse of probe light sent through the resonator in the middle of a Ramsey sequence. The Ramsey fringes rapidly decay as the atoms with different couplings to the probe field run out of phase. Note that, given our known cloud geometry and cavity parameters, we can model both this decay and the frequency of the oscillation-the differential light shift per photon traversing the cavity - and find good agreement with the data (see blue curve).

Our solution to the problem of inhomogeneous light dephasing from the probe is a form of spin echo: after the first probe pulse has traversed the cavity, we invert all atomic phases with a microwave $\pi$ pulse and then send a second probe pulse of equal energy to cancel the mean phase shift from the first pulse and rephase the atoms. The red data of Figure 3 show the 


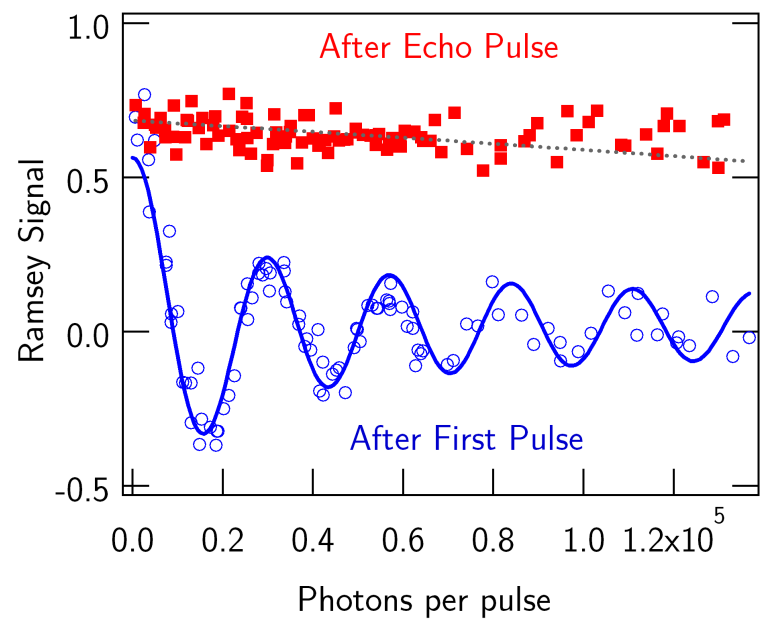

Fig. 3. Measurement of the probe light shift. A pulse of probe light is inserted into a short Ramsey sequence, measuring the mean phase imparted to the atoms by the probe photons (blue). If two probe pulses separated by a microwave $\pi$ pulse are used instead (spin echo sequence) the phase is reversed and the length of the mean spin vector becomes relatively insensitive to the probe photons.

Ramsey signal after this echo pulse. The oscillations are gone, as is most of the decay in signal amplitude. The remaining decay of around $7(1) \times 10^{-7}$ (fractional contrast loss per photon) is due to atomic motion, which changes the atoms' coupling to the probe field between the two probe pulses and thus leads to an imperfect cancellation.

Note that while the mean light shift after the two-pulse sequence is zero, we have not lost the ability to use the cavity coupling for squeezing: the two probe pulses are well separated in time and the information required for squeezing is available in the transmission difference between the two probe pulses, as the $S_{z}$ component of spin is also inverted by the $\pi$ pulse which separates them. In this respect our scheme is somewhat analogous to the technique of references [8], [12], in that two distinguishable light fields are used whose individual light shifts are large (allowing for squeezing) but whose total light shift is zero on average to cancel out most dephasing. In the case of reference [12] the fields are distinguishable by frequency, and in our implementation they are separated in time.

\section{Inhomogeneous Rabi broadening}

The coherence time of Ramsey fringes measured in our setup greatly exceeds that of Rabi oscillations. We interpret this as an indication that the Rabi frequency is inhomogeneously broadened. This is to be expected, as the atoms are located a mere $200 \mu \mathrm{m}$ from a large conducting structure; namely the chip trap used for loading the atoms into the dipole trap. The boundary conditions for the electromagnetic field at the chip and on the surrounding copper mounting structures substantially modify the intensity distribution of the microwave field on length scales comparable to the $1 \mathrm{~mm}$

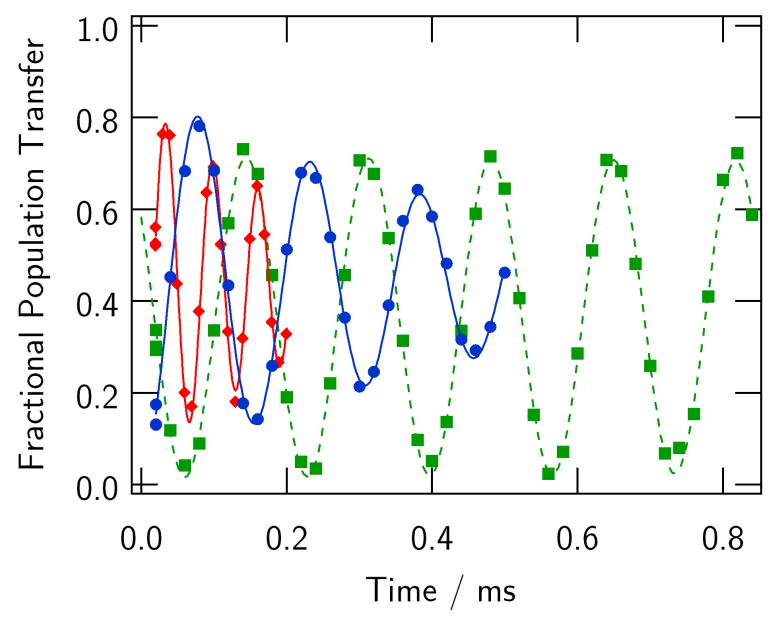

Fig. 4. Decay of Ramsey fringes (green squares) and Rabi oscillations at high (red diamonds) and low (blue circles) powers.

length of the atomic cloud. As expected for such a situation, the Rabi coherence time depends on drive power: at lower power the Rabi oscillations take a longer time, but the same number of flops, to decay (Figure 4).

An immediate practical consequence of this inhomogeneity is that the contrast of a Ramsey sequence is at best $\sim 87 \%$. That is the contrast of our best-optimized simple $\pi$ pulse, which is equivalent to the two $\pi / 2$ pulses of a Ramsey sequence.

Note that the $\pi$ pulses used in implementing the spin echo sequence are composite (SCROFULOUS [13]) pulses which correct for this inhomogeneous microwave intensity, achieving much higher contrast ( $95 \%$ ) so that our coherence is not additionally degraded by the use of spin echo. One could in principle use composite sequences for the $\pi / 2$ pulses in the Ramsey sequence, but we have not yet done so.

\section{Scattering Into Free Space}

Although our decoherence is currently limited by the technical problems described in the preceding sections, it is instructive to consider the fundamental limits imposed by photon scattering into free space. Such scattering carries two costs: it can reveal the state of individual atoms in the ensemble, and it can flip the state of individual spins.

An atom whose internal state is revealed is thereby projected into one of the two clock states and loses all coherence. For Raman scattering, which either takes the atom out of the clock subspace and into other Zeeman levels or labels its state by the color of the scattered photon, this decoherence simply occurs at the scattering rate: $\mathcal{C} \propto \exp \left(-\Gamma_{\mathrm{s}} t\right)$ where $\mathcal{C}$ is the coherence (Ramsey fringe contrast) and $\Gamma_{\mathrm{s}}$ is the rate of scattering events. Rayleigh scattering, on the other hand, does not change the atomic state and does not directly reveal it since the scattered photon has the colour of the probe light independent of the atomic state. Thus Rayleigh scattering only conveys information about the atomic state if 
the two clock states have different scattering rates. A simple calculation based on updating the density matrix of a two-level atom using Bayes' rule shows that the decoherence rate from a scattering process which happens indistinguishably (but at different rates) for the two clock states is approximately the difference between the average and geometric means of the rates: $\Gamma_{\mathrm{s}}^{\mathrm{eff}}=\frac{\Gamma_{\mathrm{s}}^{\uparrow}+\Gamma_{\mathrm{s}}^{\downarrow}}{2}-\sqrt{\Gamma_{\mathrm{s}}^{\uparrow} \Gamma_{\mathrm{s}}^{\downarrow}}$. As expected, this vanishes when the two scattering rates are equal (no information revealed) and reduces to the average scattering rate when the process occurs for only one clock state (which then gets labelled by every scattering event). For our choice of detuning, where $2 / 3$ of the scattering is Rayleigh scattering which occurs at almost equal rates for the two clock states, the decoherence rate is thus reduced to $5.5 \times 10^{-8}$ per probe photon that traverses the resonator, rather than the $1.7 \times 10^{-7}$ total scattering rate.

Interestingly, the decoherence due to scattering into free space leads to a loss of squeezing performance that is independent of atom number [12]. Thus it does not modify the fundamental scaling of the achievable signal-to-noise ratio with atom number. In contrast, Raman scattering which flips the clock spin generically reduces the achievable spin squeezing from a factor of $\sim N$ in the variance to a factor of $\sim N^{1 / 2}$ [12], [14], [15]. This is because the random flipping of spins by the scattering adds shot noise to $S_{z}$.

Since it is only spin-changing Raman scattering events that fundamentally change the scaling of the squeezing limit with optical depth, it seems promising to consider schemes where such events are forbidden. The proposal of reference [12] is one such scheme, in which each of the two clock levels is separately interrogated by a laser tuned to a cycling transition. The price paid for this reduction in spin-flip noise is a maximal loss of contrast, since each photon scattered from one of the two probe lasers unambiguously projects the scattering atom into the corresponding clock state. Ideally, one would like a system where the two clock states couple to the same laser field - so that a scattered photon does not label the state of the scatterer-but where some selection rule prevents spinchanging scattering. Such a system might allow squeezing without decoherence or noise from scattering into free space, and might potentially approach the Heisenberg limit.

\section{SQueEZING Results}

\section{A. Measurement-based Squeezing}

As described in the introduction, the shift of the resonator mode depends on the collective spin component $S_{z}$ of the intracavity atomic ensemble. A measurement of the mode shift reveals information about the ensemble spin without specifying the state of any individual atom, thus in principle preserving the coherence of the state. We perform such a measurement by driving the cavity with a probe laser detuned by one half-linewidth from cavity resonance (Figure 1c). Atom-induced shifts of the resonator frequency are revealed as changes in the transmitted intensity of this probe laser which are detected on an avalanche photodetector after the cavity.

The performace of such a measurement is shown in Figure 5. With no atoms in the cavity (green), the variance of

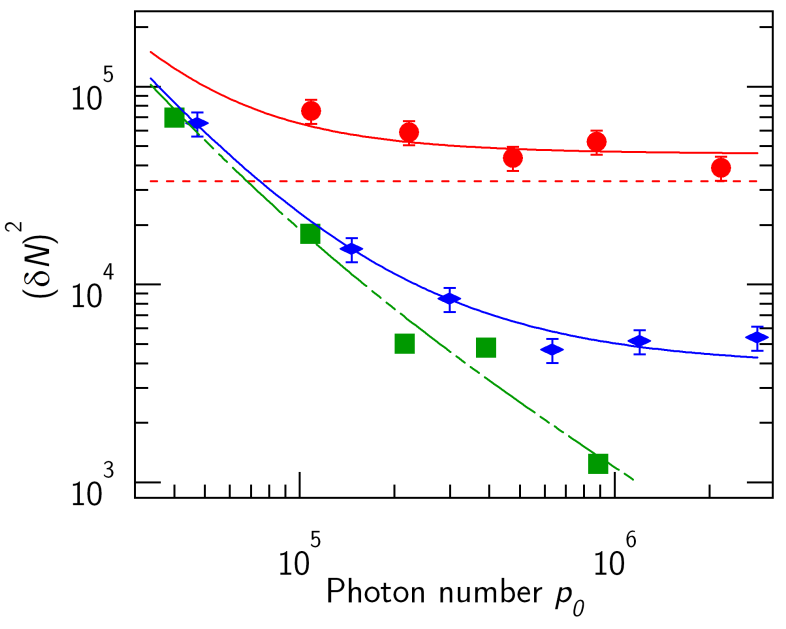

Fig. 5. $S_{z}$ measurement variance as a function of probe photon number. With no atoms in the cavity (green) we see the detection noise floor. For repeated measurements of independently-prepared uncorrelated states (red) the noise approaches the usual projection noise limit (dashed line) set by the total atom number. The variance of the difference between repeated measurements of the same atomic state (blue) is lower than this limit. This agreement between consecutive measurements indicates that the first measurement prepared a state with reduced spin noise.

the transmitted signal closely follows a model for the noise floor which includes the photocurrent shot noise augmented by our finite quantum efficiency $(50 \%)$, the excess noise of the avalanche photodiode (1.9), and a small contribution from electronic noise at small photon number. With atoms initially prepared in a coherent spin state, measured, and then reinitialized into a coherent state once again before a second measurement, we find that the variance between the measurements of the two independently prepared samples (red) approaches the standard quantum limit, as expected. If, however, we omit the intermediate optical pumping and perform the second measurement on the output state of the first measurement, we find that the two results agree much better, with a variance as much as $-9.4 \mathrm{~dB}$ below the standard quantum limit. This is what we expect for a quantum nondemolition measurement: the outcome of the first readout of $S_{z}$ should be a good predictor of the outcome of the second readout.

The quality of squeezing achieved by this QND measurement is shown in Figure 6. The blue data show our ability to predict the result of a (second) measurement of $S_{z}$ for a state that has undergone an initial squeezing measurement. The variance of the second measurement outcome relative to our prediction falls (by as much as $-9.4(8) \mathrm{dB}$ below the Standard Quantum Limit) as we use more and more photons to establish that prediction. The black curve, on the other hand, shows the contrast of a Ramsey sequence using as input the state that we prepared by the first measurement: as more and more photons are used they decohere the state until the loss of signal outweighs the reduction in noise and destroys the state's usefulness. At an intermediate photon number of $3 \times 10^{5}$ we 


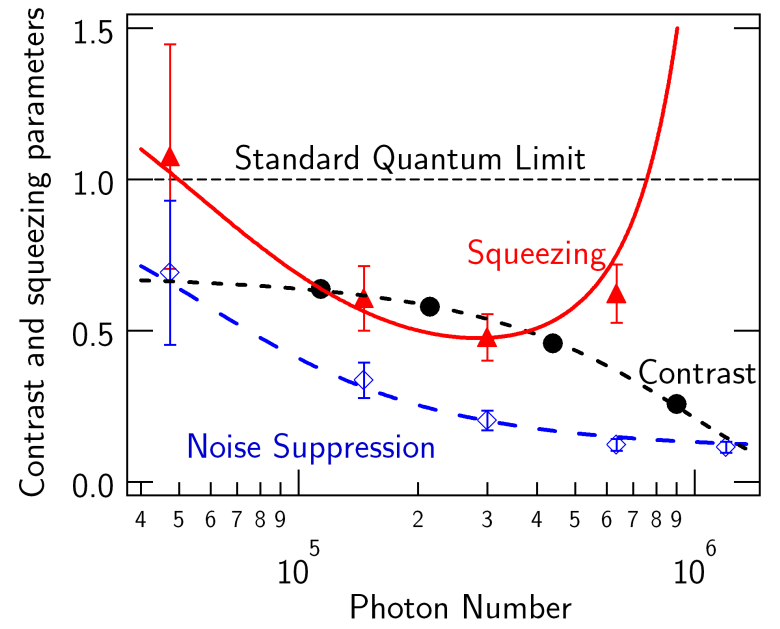

Fig. 6. Measurement-based squeezing: as more and more photons are used for the measurement of $S_{z}$, the noise on the $S_{z}$ component of the state is reduced. However the coherence of the resulting state is also reduced by inhomogeneous light phase shifts (black). Balancing the reduction of noise against the loss of signal gives the improvement in angular resolution for the collective spin (squeezing, red).

find a net squeezing [16], [17] of -3.2(8) dB, implying around a factor of two reduction in the variance of the (polar) angle of the collective spin vector.

A side benefit of implementing such a non-demolition measurement of $S_{z}$ is that it allows non-destructive readout of the atomic state so that a given sample of cold atoms can be reused, the subject of another presentation at this conference [18]. As there is almost no scattering into free space the sample does not undergo recoil heating during the measurement, and we have found that it can be reused up to 10 times, limited by the recoil heating suffered while reinitializing the spins by optical pumping. Since the cycle time is dominated by the time required to load the MOT, this is a major improvement in duty factor. All data in Section III-B were taken in this fashion, with 10 preparation and readout sequences per cooling and loading cycle.

\section{B. Deterministic Squeezing}

Rather than using information in the transmitted beam to project the atoms into a squeezed state by measurement, we can allow the atoms to evolve deterministically into such a state by exploiting their collective coupling to the resonator light field. The advantage of this method is that it is unconditional: the squeezed state produced is always the same, and so a measurement is not required. Without the need to overcome quantum efficiency and detector noise, much weaker pulses of light can be used to achieve the same noise reduction. Such weak pulses cause less decoherence (loss of signal), and so allow improved squeezing performance.

The mechanism for this squeezing is illustrated in parts (b) and (d) of Figure 1. The same $S_{z}$-dependent change in the intensity of transmitted light which was exploited for measurement in the previous subsection also leads to a change

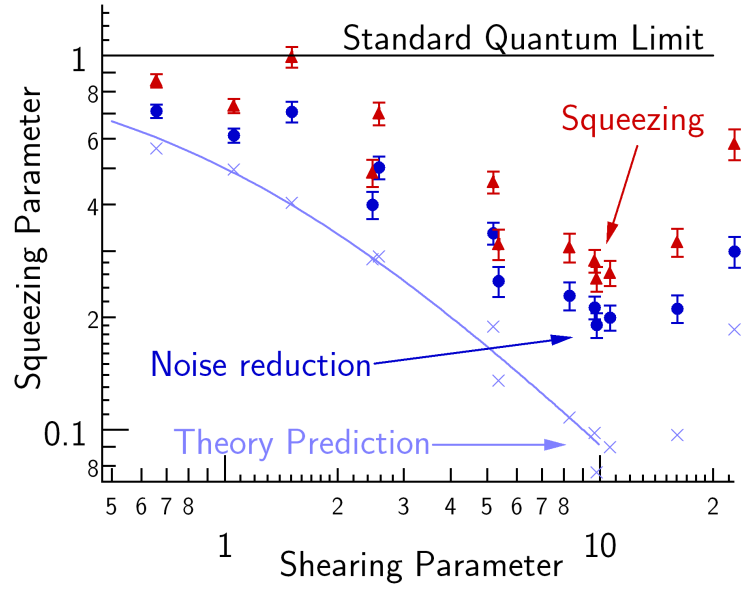

Fig. 7. Shown in blue circles are the variance of $S_{z}$ measurements for squeezed states prepared by dynamical evolution. When the loss of contrast is taken into account, we obtain the squeezing values shown as red triangles. The pale blue crosses are estimates of the intrinsic variance of the squeezed states, after subtracting out noise due to imperfect readout; they agree with a simple analytical model [19] over a decade in strength of the shearing interaction.

in the intracavity circulating intensity of the probe pulse. This change of intracavity intensity modifies the probe-induced light shift by an amount that is, to first order, linear in $S_{z}$. Thus atomic states with $S_{z}>0$ are phase-shifted more than average by the probe light, while the opposite happens for those with $S_{z}<0$. This introduces correlations between the phase shift imparted to each atom and the collective $S_{z}$ spin component, deforming the uncertainty region of the initial coherent spin state, which was circular in the $S_{y}-S_{z}$ plane, into an ellipse. The deformation is not area-preserving, as the photon shot noise on the intracavity power introduces an additional uncertainty on the amount of the phase shift. This is just the backaction of the measurement we could have performed by measuring the transmitted light. Nonetheless, the resulting uncertainty distribution is narrower in some diagonal direction than the original coherent state, and a microwave rotation about the mean spin axis can convert this into a reduction in variance of the spin projection $S_{z}$.

Figure 7 shows the reduction in noise achieved at the optimum rotation angle for various strengths of this shearing effect, which depends on both the photon number sent through the cavity and on the atom number [19]. Although the noise reduction is not as great as the measurement-induced noise suppression of Figure 6, it occurs for a much lower photon number: typically $6 \times 10^{4}$ instead of $3 \times 10^{5}$. As expected, the reduced decoherence afforded by such weak squeezing pulses dramatically improves the contrast (from $50 \%$ to $80 \%$ for the best squeezing) and thus we reach $-6.0(4) \mathrm{dB}$ of squeezing.

\section{CONCLUSION}

We have discussed some of the decoherence mechanisms, both technical and fundamental, that limit squeezing perfor- 
mance and briefly presented the performance that we achieve in spite of those limitations. Encouragingly, all the currently relevant limitations are technical: for our parameters the fundamental limit from photon scattering into free space allows as much as $-18 \mathrm{~dB}$ of squeezing [9], [14], [15], so that there is considerable room for further improvement.

The squeezing schemes we have demonstrated are not restricted to microwave-transition clocks, where both clock levels are near-resonant with the same optical transition. A general and straightforward method for extending these tools to optical clocks is to couple the cavity to only one of the two clock levels and use a $\pi$ pulse to exchange population between coupled and uncoupled states. Provided the $\pi$ pulse fidelity is sufficiently high, this sequential interaction with the two populations is enough to implement all the squeezing techniques presented here. Other, more elegant and robust schemes may also be available for atoms with specific level structures.

Finally, an important question is the extent to which, and the circumstances under which, squeezing is beneficial to the performance of real-world clocks. It has been shown that clocks whose Ramsey precession time is limited by atomic decoherence do not substantially benefit from the use of squeezed input states [20], while those clocks limited by local oscillator noise can expect some performance improvement [21]. Clocks that have an externally imposed Ramsey time, set for instance by the requirement of frequent readouts to increase the bandwidth of a servo loop, could see the full reduction in phase noise promised by squeezing, while others may have richer noise models than those considered in theoretical studies so far. Now that metrologically interesting quantum correlations can be generated, the problem of using them in practical measurement devices is ripe for further study.

\section{ACKNOWLEDGEMENT}

We thank J.K. Thompson, M.D. Lukin, D. Stamper-Kurn and E.S. Polzik for fruitful discussions. This work was partially funded by the NSF, DARPA and the Center for Ultracold Atoms. M.H.S. acknowledges support from the Hertz Foundation under the Daniel Strook fellowship and I.D.L. acknoledges support from NSERC.

\section{REFERENCES}

[1] G. Santarelli, P. Laurent, P. Lemonde, A. Clairon, A. G. Mann, S. Chang, A. N. Luiten, and C. Salomon, "Quantum projection noise in an atomic fountain: A high stability cesium frequency standard," Phys. Rev. Lett., vol. 82, p. 4619, 1999.

[2] M. Kitagawa and M. Ueda, "Squeezed spin states," Phys. Rev. A, vol. 47, p. $5138,1993$.

[3] J. Hald, J. L. Sørensen, C. Schori, and E. S. Polzik, "Spin squeezed atoms: A macroscopic entangled ensemble created by light," Phys. Rev. Lett., vol. 83, p. 1319, 1999.

[4] V. Meyer, M. A. Rowe, D. Kielpinski, C. A. Sackett, W. M. Itano, C. Monroe, and D. J. Wineland, "Experimental demonstration of entanglement-enhanced rotation angle estimation using trapped ions," Phys. Rev. Lett., vol. 86, pp. 5870-5873, 2001.

[5] J. Estéve, C. Gross, A. Weller, S. Giovanazzi, and M. K. Oberthaler, "Squeezing and entanglement in a bose-einstein condensate," Nature, 2008, advance online publication. [Online]. Available: http://www.nature.com/nature/journal/vaop/ncurrent/abs/nature07332.htm

[6] S. Chaudhury, S. Merkel, T. Herr, A. Silberfarb, I. H. Deutsch, and P. S. Jessen, "Quantum control of the hyperfine spin of a Cs atom ensemble," Phys. Rev. Lett., vol. 99, p. 163002, 2007.

[7] T. Fernholz, H. Krauter, K. Jensen, J. F. Sherson, A. S. Sørensen, and E. S. Polzik, "Spin squeezing of atomic ensembles via nuclear-electronic spin entanglement," Phys. Rev. Lett., vol. 101, p. 073601, Aug. 2008.

[8] J. Appel, P. Windpassinger, D. Oblak, U. Hoff, N. Kjaergaard, and E. S. Polzik, "Quantum noise squeezing and entanglement on the atomic clock transition," 2008, arxiv:quant-ph/0810.3545.

[9] M. H. Schleier-Smith, I. D. Leroux, and V. Vuletić, "Reduced-quantumuncertainty states for an atomic clock," 2008, arxiv:quant-ph/0810.2582.

[10] I. D. Leroux, M. H. Schleier-Smith, and V. Vuletić, "Spin squeezing by one-axis twisting: Entangling a dilute atomic ensemble by dynamical evolution," 2009, to be published.

[11] I. H. Deutsch and P. S. Jessen, "Quantum-state control in optical lattices," Phys. Rev. A, vol. 57, no. 3, pp. 1972-1986, Mar 1998.

[12] M. Saffman, D. Oblak, J. Appel, and E. S. Polzik, "Spin squeezing of atomic ensembles by multi-colour quantum non-demolition measurements," 2008, arxiv:quant-ph/0808.0516.

[13] H. K. Cummins, G. Llewellyn, and J. A. Jones, "Tackling systematic errors in quantum logic gates with composite rotations," Phys. Rev. A, vol. 67, no. 4, p. 042308, Apr 2003.

[14] K. Hammerer, K. Mølmer, E. S. Polzik, and J. I. Cirac, "Light-matter quantum interface," Phys. Rev. A, vol. 70, p. 044304, 2004.

[15] L. B. Madsen and K. Mølmer, "Spin squeezing and precision probing with light and samples of atoms in the gaussian description," Phys. Rev. A, vol. 70, p. 052324, 2004.

[16] D. J. Wineland, J. J. Bollinger, W. M. Itano, F. L. Moore, and D. J. Heinzen, "Spin squeezing and reduced quantum noise in spectroscopy," Phys. Rev. A, vol. 46, p. R6797, 1992.

[17] D. J. Wineland, J. J. Bollinger, W. M. Itano, and D. J. Heinzen, "Squeezed atomic states and projection noise in spectroscopy," Phys. Rev. A, vol. 50, p. R67, 1994.

[18] J. Lodewyck, P. Westergaard, A. Lecallier, and P. Lemonde, "Nondestructive measurement of the transition probability in a ${ }^{87} \mathrm{Sr}$ optical lattice clock," 2009, lecture at EFTF-IFCS Joint Conference in Besan con, France.

[19] M. H. Schleier-Smith, I. D. Leroux, and V. Vuletić, "Squeezing of the collective spin of an atomic ensemble by cavity feedback," 2009, to be published.

[20] S. F. Huelga, C. Macchiavello, T. Pellizzari, A. K. Ekert, M. B. Plenio, and J. I. Cirac, "Improvement of frequency standards with quantum entanglement," Phys. Rev. Lett., vol. 79, p. 3865, 1997.

[21] A. André, A. S. Sørensen, and M. D. Lukin, "Stability of atomic clocks based on entangled atoms," Phys. Rev. Lett., vol. 92, p. 230801, 2004. 